the well-being of one nation at the cost of another, the tendency to value one's own civilization as higher than that of the whole race of mankind, are the same as those which prompt the actions of primitive man, who considers every stranger as an enemy, and who is not satisfied until the enemy is killed. It is somewhat difficult for us to recognize that the value which we attribute to our own civilization is due to the fact that we participate in this civilization, and that it has been controlling all our actions since the time of our birth; but it is certainly conceivable that there may be other civilizations, based perhaps on different traditions and on a different equilibrium of emotion and reason which are of no less value than ours, although it may be impossible for us to appreciate their values without having grown up under their influence. The general theory of valuation of human activities, as taught by anthropological research, teaches us a higher tolerance than the one which we now profess.

Our considerations make it probable that the wide differences between the manifestations of the human mind in various stages of culture may be due almost entirely to the form of individual experience, which is determined by the geographical and social environment of the individual. It would seem that, in different races, the organization of the mind is on the whole alike, and that the varieties of mind found in different races do not exceed, perhaps not even reach, the amount of normal individual variation in each race. It has been indicated that, notwithstanding this similarity in the form of individual mental processes, the expression of mental activity of a community tends to show a characteristic historical development. From a.comparative study of these changes among the races of man is derived our theory of the general development of human culture.
But the development of culture must not be confounded with the development of mind. Culture is an expression of the achievements of the mind, and shows the cumulative effects of the activities of many minds. But it is not an expression of the organization of the minds constituting the community, which may in no way differ from the minds of a community occupying a much more advanced stage of culture.

\section{Franz Boas.}

\section{ASSOCIATION OF AMERICAN ANATOMISTS.}

The fourteenth session of the Association of American Anatomists, meeting with the American Society of Naturalists and Affiliated Societies in Baltimore, Md., was held in the Anatomical Laboratory of the Johns Hopkins University, December 27 and 28, 1900.

The meeting was called to order, December 27th at 10:20 A.M., by President George S. Huntington.

The Executive Committee reported and recommended the names of eleven candidates for membership. Also a recommendation that at the discretion of the secretary the first five 'Proceedings,' now out of print, should be reprinted. Also a recommendation that the Association endorse the proposition for the establishment of a psycho-physical laboratory in the Bureau of Education, Washingtón, D. C.

By unanimous consent the secretary cast the ballot for the nominees for membership. The Association also authorized the secretary to reprint the five 'Proceedings' as recommended. The recommendation to endorse the psycho-physical laboratory was not agreed to and was referred to a committee to be appointed by the president to report at a future meeting. It was discussed unfavorably by Drs. Holmes and Hrdlicka.

The Secretary made his yearly report, which stated, among other things, that he had in hand copies of the 'Proceedings' 
of the Association from the 6th to the 13th meeting, which were available for distribution to members and especially to libraries. There were 33 libraries and journals on the regular mailing list. The financial exhibit showed a balance in the treasury of $\$ 177.47$; total receipts $\$ 492.25$; expenditures $\$ 314.78$. He suggested the desirability of having a summer meeting with the American Association for the Advancement of Science. The Association had lost several members since May, 1900, when a provisional report had been made. Professor Wm. Anderson of London, an honorary member, died Oct. 27th. Dr. A. L. T. Schäper, of Harvard Medical School, had left this country, having been appointed a professor at the University of Breslau, Prussia. There were now 116 active and 9 honorary members, total, 125.

No reports were received from the delegate to the executive committee of the Congress of American Physicians and Surgeons, nor from the committee on anatomical peculiarities of the negro, nor from the committee on table at Naples. The committee on anatomical nomenclature reported prog. ress.

The President appointed a committee consisting of Drs. Huber, Carmalt and Barker to report nominations for delegates to the executive com mittee of the Triennial Congress and a new member of the executive committee of this Association.

Dr. Huntington then read the Presidential address, taking for his subject 'The Morphological Museum as an Educational Factor in the University System.'

The following papers were read:

\section{The Use of Wet Specimens: Dr. Holmes,} Philadelphia.

The cry of the general public for practicability and the desire of the recent graduate for rapid success, and strangely enough the theory of our medical schools, 'science for its own sake,' are all tending to the same point, namely, the training of students to be scientists before they have been educated as physicians. As a resultant of these forces, our medical schools are getting away from their original intent of turning out practising physicians and are evolving onesided specialists, which again, strangely enough, the tendency to laboratory and section teaching only seems to increase, by compelling men to choose certain subjects to the exclusion of others. Following this has come a disinclination to instruct and a neglect of teaching method, so that, as has been said in anatomy, 'a man who has a book, a subject and a scalpel, ought to be able to work it all out for himself.' The most obvious improvement in our branch is the teaching in small sections; next the methodical daily apportionment of the work. If we could have our way, we would not only assign the same dissection for the same hour, but if it were possible we would have our scalpels ply together with the same unison as the violin bows in a well-trained orchestra. To be ahead of the assignment is a crime, to be behind it far better, if it implies not sloth nor ignorance, the most careless students being the most rapid slashers. Methodical and clean dissection implies a foreknowledge of the structure, but it is difficult to impress the fact that the dissecting room is a laboratory and not a library alcove, didactic reading should be done at home, the only use of the book being in connection with the cadaver. Explanations should be from the wet specimen and not merely a worded exposition. The wet specimen of muscle, artery or joint should be kept continually before the student as a pattern, to supply the defects of his own work, to study the deeper connections or to review the more superficial which must necessarily be cut away and, at the conclusion of the dissection, for a review of the whole part. Equally for the alumnus 
dissector whose time is limited. With a book upon applied anatomy such hurried practitioners with a dissected subject before them can get ten times more practical benefit than could possibly be accomplished by an individual not an expert. If we were given a choice with the average man between a course of study upon the cadaver with carefully prepared wet specimens and the hacked up dissection, we should withont hesitation recommend the former. For intelligent comprehension, based on sound pedagogical principles, instruct your student, first, as to what to find, and where, ' in the wet,' and then careful, neat, systematized dissection can not be done too often. For the preservation of wet specimens cold storage is by far the best, with the ' Kaiserling' next. Alcohol hardens them too much, solutions of chloral waterlog them, formalin preparations favor mold of any part from which the fluid is allowed to evaporate.

Dr. Corson, of Savannah, Ga., not being able to be present, his paper, 'The Value of the X-ray in the Study of Normal Anatomy,' illustrated by photographs of the human membral epiphyses at the thirteenth year, was read by Dr. Kerr of Cornell University. The paper contended that the $\mathrm{X}$-ray would prove of value, first, in the study and demonstration of bone development the growth of the epiphyses, the schema of their development and the study of joints as joints, with their movements ; second, in the demonstration of the internal structure of the bones; third, in the study and demonstration of the exact spacings and positions of the bones in the skeleton as a guide to its proper articulation and mounting; this would find its widest application in comparative osteology ; fourth, in the study and demonstration of the arteries on the cadaver, where properly injected, they can be skiagraphed in their absolute relations to other structures. The possibility of this work is wholly due to great improvements in apparatus and technique, and without doubt we can look for even greater improvements in the future in X-ray intensity, which will widen its present field of usefulness. By the.X-ray we can really watch the bones grow, and we get certain projected plans of bones and their exact positions in the skeleton which give us new ideas of function as well as of form. Thus physiology as well as morphology will benefit by the discovery of Röntgen.

The Levator Ani Muscle: Dr. HoLmes, Philadelphia.

The levator ani muscle arises internal to the obturator fascia, on a line from the posterior surface of the crest of the pubes to the spine of the ischium; the fibrous leaf-lets, projecting proximal and distal to the muscle, and running downwards and inwards parallel to its fibers, being called the rectovesical and anal fascias, which for our purpose form a sheath for the levator ani, but in reality constitute the true supporting floor of the pelvic outlet. The levator ani muscle in its origin is unique. At its in. sertion it is fixed only at the perineal center and the coccyx, while at the median raphe it is movable throughout, though counterbalanced by its fellow of the opposite side; and at the sphincters it is as yielding as the soft viscera themselves. None of the fibers are attached to the prostate gland, though they go behind it to join with the opposite muscle constituting a compressor as well as a levator prostatæ; so to the sphincters of the anus and of the vagina we can trace the muscular fibers, but not to the walls of the vagina nor of the rectum. The rectovesical fascia which forms the proximal side of the muscular sheath blends with the fibrous coat of each canal, but the only direct interlacement of the muscular fibers is with the sphincters. 
It would seem, therefore, as if the authors were in error in asserting that the levator ani is inserted 'into the lateral aspect of the prostate,' 'into the side of the rectum' or 'into the walls of the vagina,' but as in its origin, the muscles are attached to a narrow linear movable insertion.

It is more a tensor of the fascia, either at its origin or insertion, the fixed point being interchangeable, so that it should be called the 'Tensor perineæ' rather than ' Levator ani.'

Development of the Human Diaphragm: DR. Mall, Baltimore. (Illustrated by diagrams and specimens.)

In human embryos four weeks old the pericardial and peritoneal cavities freely communicate. At this time, however, a ridge of tissue is formed in the wall of the colom opposite the ductus Cuvieri and cardinal veins which grows rapidly and encircle the lungs to form the pulmonary ridge. A week later the ridge widens to form the beginning of two membranes. The first of these, the pleuro-pericardial membrane, contains within it the phrenic nerve and soon separates the pleural from the pericardial cavity. The second, the pleuro-peritoneal membrane, grows towards the tail with the rotation of the liver and the degeneration of the Wolffian body, and at the end of the sixth week completes the diaphragm.

Dr. Stroud, of Cornell University, was unable to be present. A photograph sent by him, showing apparatus for demonstrating the circulation of the blood, was passed around among the members.

The apparatus is an imitation of the actual blood vascular system. The heart, arteries, capillaries and veins are represented by a rubber bulb with valves, very elas. tic rubber tubing; capillary glass tubing and thinner rubber tubing. The bifurcation of arteries is shown in $\mathrm{Y}$-shaped, of veins by U-shaped, glass tubes. The circulation is continuous as in the living body. Manometer tubes indicate the difference in pressure in arteries and veins.

Advantages and Limits of the Methods of Reconstruction with Wax Plates in Anatomical and Embryological Investigations. Dr. CHAs. R. Bardeen, Baltimore. (Illustrated by specimens, etc.)

Discussed by Drs. Huber, Minot, Barker, W. S. Miller and Huntington.

Demonstration of a New Freezing Microtome : Dr. BARDEEN.

Specimen of Cyclopia: Dr. Carmalt, New York City. (With cast and photographs.) Discussed by Dr. Minot.

A Caudal Appendage in a Human Infant: Dr. Harrison, Baltimore. (Illustrated by specimen and photographs.)

Discussed by Dr. Hrdlicka. A tail-like appendage, measuring $2 \frac{1}{4}$ inches in length, was present in an infant of four months. The tail arose close to the tip of the coccyx, although not a direct continuation of the latter. It contained no cartilage nor bone, but did contain voluntary muscle fibers, and was movable to a considerable degree.

Typical Forms of Shaft of Long Bones other than the Tibia: Dr. HrdlickA, New York City. (Illustrated by specimens.)

Discussed by Drs. Huber and Huntington. The paper presents the further results of the writer's investigations on Professor Huntington's osteological collection in the Medical Department of Columbia University, New York City. It deals with the variations in shape of long bones and classification of these shapes in a similar manner as the former (1898) communication on the tibia. An inference will be drawn as to the causes of the variation.

Notes on the First and Second Ribs and a Demonstration of Bicipital, Bicaudal, Notched and Perforated Ribs in Man; also Notes and 
Articulation of Ribs with each other: DR. Hrdlick. (Illustrated by specimens.) Discussed by Dr. Huntington. Variations in shape of normal first and second ribs; the scalene tubercle, its frequency and real nature, and a similar tubercle on the second rib; peculiarity of the normal ossification of the cartilage of first rib. Demonstration of anomalous specimens.

Variations of the Inferior Cava: DR. HuntINGTON. (Illustrated by photographs.)

The Origin of the Lymphatics of the Liver : DR. MaLL.

When Prussian-blue gelatin in which cinnabar is suspended is injected into either the portal or hepatic vein, it is found that the blue filters through the capillary wall, leaving the red granules in the capillaries. The capillaries are surrounded by the perivascular lymph spaces which communicate with perilobular lymph spaces. These, in turn, communicate freely with the interlobular lymphatics.

The Lobule of the Lung : DR. W. S. MrLLER, Madison, Wis. (Illustrated by models, diagrams and lantern slides.)

Discussed by Drs. Huntington and Huber. The term lobule as applied to the unit of the lung has been used in an exceedingly vague sense both by anatomists and pathologists. It is the purpose of the paper to give a definite meaning to the term.

The Epithelium of the Pleural Cavities: Dr.

MrLLer, Madison, Wis. (Illustrated by lantern slides and preparations.)

Since the time of v. Recklinghausen and Oedmansson certain dark spots seen in many preparations of serous membranes stained by the silver nitrate method have been called stomata and stigmata. Ludwig and Dybkowsky described such structures in the pleura. Muscatello has recently shown that such openings do not exist normally in the peritoneum. It is the pur- pose of the paper to show that they do not exist in the pleura when studied in the normal condition, and that they can be produced artificially at the pleasure of the investigator.

Preliminary Report with Projection Drawings, illustrating the Topography of the Paracoles in their Relation to the Surface of the Cere'brum and Cranium: Mr. E. A. SPITzkA, New York City. (Illustrated by drawings and diagrams.)

Since the tapping and injecting into the ventricles have become definite procedures in surgery, it would greatly aid the operator to have a more accurate conception of the extent, depth and contour of the cavities, with their variations, than can be had from the bare rules and measurements set forth in most surgeries. With this view the author utilizes the entire head, hardened by injection of, and submersion in, formal. After a time the cranium is opened and the brain is accurately sliced, correct drawings being made at each step and projected for the delineation of the final plates. Two heads have so far been completed. It is proposed to decalcify the skulls of subsequent material, the entire head being then subjected to the slicing method. [Published in New York Medical Journal, February, 2, 1900.]

Bilateral Relations of the Cerebrat Cortex : Dr. Mellus, Baltimore.

Hastily reviewing bilateral relations previously demonstrated, he called attention to a series of his recent experiments on the monkey. After extirpation of a small portion of cortex from that part of the so-called motor area situated on the boundary line between facial and upper limb centers, he showed degenerated fibers passing from the lesion across the middle line in the corpus callosum. These fibers were distributed to cortical areas of the opposite hemisphere corresponding to the convolutions upon the 
side of the lesion, to which association fibers were traced directly from the lesion. This distribution of association fibers to the convolutions of the two hemispheres was quite symmetrical, and by far the greater number passed to that portion of the opposite hemisphere which corresponded to the situation of the lesion. In addition to these association fibers a large portion of the degenerated tract crossing in the corpus callosum turned down into the internal capsule of the opposite side. These fibers mostly terminated in the thalamus and hypothalamus, but in a number of instances a few of these fibers passed on through the pons and medulla into the cord. Owing to the degeneration in both pyramids above the decussation, it could not be determined whether or not these fibers recrossed in the decussation. Dr. Mellus stated that after unilateral lesions in the brain of the dog he had found fibers crossing in the corpus callosum and passing down the internal capsule of the opposite side. In no instance, however, had he found these fibers in the dog passing through the capsule. They all passed out of the capsule and ended in the thalamus of the side opposite the lesion.

Methods of Statistical Study in the Dissecting Room with special reference to the Peripheral Nervous System: Dr. BARDEen. (Illustrated by charts.)

Discussed by Dr. Huntington.

Wandering of the Skin during Development, in Relation to the Distribution of Cutaneous Nerves : Dr. Harrison. (Illustrated by diagrams.)

By a process of transplantation, embryos of two species of frogs may be combined, and through contrast in pigmentation, wandering of the epidermis may be accurately observed as the embryo develops. There is a definite relation between this movement and the course of the cutaneous nerves in the adult. The wandering of the skin is, at least to a great extent, passive, and is due to mechanical causes.

\section{Intrinsic Blood Vessels of the Kidney and their} Significance in Nephrotomy: MR. Max BröDEL, Baltimore.

The pelves of most kidneys are on the posterior part of the hilum and pass obliquely forward to the center of the kidney. The calices are grouped in two rows, anterior, pointing forward, and a posterior, pointing laterally. The arteries usually divide into two main trees, an anterior, carrying three-fourths to four-fifths of the blood, and a posterior furnishing the remainder. There is neither anastomosis nor crossing between the branches of these two trunks; they are completely separated by the pelvis and its calices. The superficial and deep collecting veins of the cortex empty into the venous arches at the base of the pyramids, forming the peripheral system of venous anastomoses. There is, however, a second more central system around the necks of the calices. Practically all the veins at the hilum pass anteriorly to the pelvis. The form of the pyramids and their relations to the blood vessels and surface, and also the columns of Bertini, have been studied. Although many kidneys deviate in their construction, all conform more or less to the rules mentioned. The studies reveal several points of interest to the surgeon, among which are: (1) The most advantageous incision for nephrotomy. (2) The appropriate method of suturing an incised kidney. (3) A very satisfactory method of fixing the kidney. (4). The insufficiency of the method of opening the pelvis at the hilum.

Histology of the Endometrium : DR. T. S. CuLLEN, Baltimore. (Illustrated by specimens and drawings.)

The Architecture of the Gall-bladder: Dr. M. T. Sudler, Baltimore. (Read by title.) 
The Classification of Glainds: Dr. Mrnot, Boston. (Read by title.)

Contribution to the Question of Fissural Integrality of the Paroccipital; Observations on 100 Brains: Mr. Spitzka. (Illustrated by drawings.)

Examination of 100 brains in the anatom ical laboratory of the College of Physicians and Surgeons, New York City, yielded results similar to those of Professor Wilder. The question of the integrality, with the hypotheses of Cunningham, Wilder and Parker, are briefly discussed. The writer inclines to the opinion that it is not an integer, but a partial and modified segment of the simian exoccipital; and, further, that its confluence with, or separation from, the parietal should be regarded as o secondary significance and importance.

The Mesial Relations of the Inflected Fissure.

Observations on 100 Brains: Mr. Spitzka.

(With about 60 illustrations.)

The chief points are: (1) the normal position of the inflected fissure on the meson is caudad of the cephalic limb of Wilder's paracentral fissure in other words, its mesial portion indents and partly lies within the paracentral lobule (or as Broca prefers to call it, ' oval lobule'). (2) There is considerable confusion in modern ehcephalic literature concerning the synonymy of the inflected fissure. The most erroneous statements come from Eberstaller, and consist in a misinterpretation of Broca's 'incisure pré-ovalaire' and Schwalbe's 'sulcus paracentralis,' with the 'inflected fissure' of Lussana and Wilder, ' $X$ fissure' of Benedict and Flesch, or 'sulcus præcentralis medialis' of Eberstaller and other European writers. Broca's and Schwalbe's fissure corresponds to the cephalic limb or limiting ramus of Wilder's paracentral. (3) The condition presented in the mulatto brain described by Wilder (see Figs. '4766 and 4772 of his article,
Handbook, Vol. VIII., 1889) is anomalous and rare, since the inflected appears wholly without the paracentral gyrus, and therefore cephalad of Broca's 'pre-oval incisure' or Wilder's cephalic paracentral limb. The explanation appears to be that the cephalic limb is obliterated, while simultaneously one of the intraparacentral elements, of which there seem to be several, has effected a junction with the paracentral stem, and thus appears, at first glance, to be a well-defined cephalic ramus.

The Brains of Two Distinguished Physicians, Father and Son; a Comparative Study of their Fissures and Gyres: Mr. Spitzka. (Illustrated by drawings and photographs.)

Discussed by Drs. Lamb and Huntington . The paper 1 ats carefully and at length of the brains o. Jr. Edouard Seguin and his son, Dr. Edward C. Seguin. The learning and progressiveness of these men are well known in the educational and scientific world. Both were interested in the study of idiocy and medical thermometry; the father was especially instrumental in introducing the metric system into this country, while the son, as one of the pioneers of American neurology, made many valuable contributions to the pathology and therapeutics of nervous diseases. The brains show a general similarity and refined development, but with differences as puzzling as profound ; and many interesting features appear in the comparison. So far as the author knows, this is the first instance in which the brains of blood relatives have been compared and described.

\section{Method of .Utilizing Frozen Sections for Class} Demonstrations of Visceral Anatomy and the Epiphyses: Professor Primrose, Toronto, Canada.

The exhibition of lantern slides was a series of photographs made from sections through the trunk and extremities of chil- 
dren. The sections were prepared in a special manner so as to present a perfectly smooth surface with clear outlines of the various. structures. These sections were photographed and lantern plates made from the negatives. They were cut in sagittal, coronal and horizontal planes through the trunk, and in longitudinal and transverse directions through the extremities. The method adopted in the University of Toronto is that permanent preparations are made of the sections, which are mounted in flat dishes, and thus exposed, so that they are accessible for the students at any time in the Anatomical Department. The lantern demonstration of these sections is given from time to time at the close of a lecture. It proves to be a very useful adjunct to the ordinary methods of demonstration, and the student always has the opportunity of studying the actual section in the dissecting room, the photograph of which is thrown upon the screen in the lecture theater. It is claimed that these photographs of actual sections are of much greater value from an educational standpoint than the drawings reproduced from the sections.

Method of Teaching the Anatomy of the Central Nervous System to Large Classes of Students : Dr. Barker, Chicago, Ill. (Read by title.)

\section{S. LAMB,} Secretary.

THE ELEVENTH MEETING OF THE AMERICAN MORPHOLOGICAL SOCIETY.

THE American Morphological Society held its eleventh annual meeting in the Anatomical Laboratory of Johns Hopkins University, on the 27th and 28th of last December. A good proportion of members was present.

The following officers were elected for the present year : President, J. S. Kingsley ; Vice-President, E. A. Andrews: SecretaryTreasurer, Thos. H. Montgomery Jr. ; Mem- bers of the Executive Committee, C. F. W. McClure and C. W. Hargitt. Twelve new members were elected; and the Society voted fifty dollars for the support of the University table at the Naples laboratory.

The following papers were read (abstracts of which will be published in the Biological Bulletin):

Fission and Regulation in Stenostoma leucops : C. M. CHILd.

On Gunda segmentata in America: W. C. CurTis.

Exhibition of Pacific Coast Nemerteans: W. R. CoE.

Some Disputed Points in the Anatomy of Limpets : M. A. Willcox.

The Habits and Life History of Argulus, with reference to its Economic Relations: C. B. WILson.

A Comparative Study of the Development of the Germinative Tract of Termites: H. MoE. KNOWER.

The Anatomy and Development of the Vena cava in Didelphys Virginiana: C. F. W. MoClure.

The Crossing of the Optic Nerves in Teleosts : G. H. Parker.

A New Type of Budding in Annelids : H. P. JoHnson.

Amphibian Studies: J. S. Kingsley.

Phagocytosis in a Mammalian Ovary: M. M. Metcalf.

The Mammalian Lower Jaw : W. H. RudDick and J. S. KINGSLEY.

An Apparatus in the Central Nervous System of Vertebrates for the Transmission of Motor Reflexes arising from Optical Stimuli: P. E. SARGENT.

The Structure of the Testis in Desmognathus fuscus: B. F. Kingsbury.

The Synapsis Stage of the Germ Cells : T. H. Montgomerí, JR. 